\title{
Ion-Specific Self-Assembly of Hydrophobically Modified Polycation of Natural Origin
}

\author{
Evgeniya V. Korchagina and Olga E. Philippova* \\ Physics Department, Moscow State University, Moscow 119991, Russia, e-mail: \\ phil@polly.phys.msu.ru
}

\section{SUPPORTING INFORMATION}

Treatment of Light Scattering Data. Static light scattering (SLS) experiments were performed to determine the second virial coefficient $A_{2}$, the apparent weight-average molecular weight $M_{w}{ }^{*}$, and the apparent z-average radius of gyration $R_{g}{ }_{g}$. In these studies, the absolute scattered intensity, $R_{\theta}(q)$ (excess Rayleigh ratio), of the solute particles is determined from the experimentally measured scattered intensities of the solution, $I_{\text {solution }}$, and of the solvent, $I_{\text {solvent }}$, as well as the intensity measured for a toluene sample standard, $I_{\text {toluene, }}$, renormalized by the socalled absolute scattering intensity of the standard $I_{\text {toluene, abs }}:{ }^{1}$

$$
R_{\theta}(q)=\left(I_{\text {solution }}-I_{\text {solvent }}\right) \frac{I_{\text {toluene }, a b s}}{I_{\text {toluene }}}
$$

The SLS experiments were carried out at scattering angles ranging from $30^{\circ}$ to $150^{\circ}$.

\footnotetext{
* To whom correspondence should be addressed.
} 
To account for the effects of the particle concentration, the particle form factor, and solutesolvent interactions on the measured scattering intensity the Zimm expression ${ }^{2}$ was used:

$$
\frac{K c}{R_{\theta}(q)} \approx \frac{1}{M_{w}}\left(1+\frac{1}{3} R_{g}^{2} q^{2}\right)+2 A_{2} c
$$

where $c$ is the polymer concentration, $K=4 \pi^{2} n^{2}(d n / d c)^{2} / N_{A} \lambda^{4}$ is the scattering constant, and $q=4 \pi n / \lambda \sin (\theta / 2)$ is the scattering vector. Here $N_{A}$ is Avogadro's number, $\lambda$ is the wavelength of light in a vacuum, $n$ is the refractive index of solvent, and $d n / d c$ is the refractive index increment. According to eq $\mathrm{S} 2$, the extrapolation $\left(K c / R_{\theta}\right)_{c \rightarrow 0, q \rightarrow 0}$ gives the $1 / M_{w}$ value, whereas the slopes of $\left(K c / R_{\theta}\right)_{c \rightarrow 0}$ vs $q^{2}$ and $\left(K c / R_{\theta}\right)_{q \rightarrow 0}$ vs $c$ lead to $R_{g}$ and $A_{2}$ values, respectively.

Dynamic light scattering (DLS) measurements were carried out in order to determine the hydrodynamic radii, $R_{H}$, of scattering particles. The scattering angle $\theta$ was varied between $30^{\circ}$ and $90^{\circ}$. In DLS experiments the normalized time autocorrelation function, $g^{(2)}(q, t)$, of the scattered intensity is measured and expressed in terms of the field autocorrelation function, $g^{(1)}(q, t)$ (or the autocorrelation function of the concentration fluctuations), through the equation ${ }^{2}$

$$
g^{(2)}(q, t)=\frac{\langle I(q, t) I(q, 0)\rangle}{\langle I(q, 0)\rangle^{2}}=A+\beta\left|g^{(1)}(q, t)\right|^{2}
$$

where $I(q, 0)$ and $I(q, t)$ are the scattering intensities at time $t=0$ and at a certain delay time $t$ later, $A$ is the baseline, and $\beta$ is the coherence factor.

The distribution of decay rates (reciprocal relaxation times), $G(\Gamma)$, was determined from the correlation function $g^{(l)}(q, t)$ using the CONTIN algorithm. ${ }^{3}$ It is based on the Laplace inversion of $g^{(I)}(q, t)$ function. When the spectral profile of the scattered light can be described by a multiLorentzian curve, $g^{(l)}(q, t)$ can be written as ${ }^{2}$

$$
g^{(1)}(q, t)=\int_{0}^{\infty} G(\Gamma) \exp (-\Gamma t) d \Gamma
$$


In the case of a diffusive process, $\Gamma$ is related to the translational diffusion coefficient $D_{0}$ by $D_{o}=\left.\left(\frac{\Gamma}{q^{2}}\right)\right|_{q=0, c=0}, \quad$ so that $G(\Gamma)$ can be converted into a translational diffusion coefficient distribution or a hydrodynamic radius distribution by using the Einstein - Stokes equation. ${ }^{2}$

The gyration radii of the aggregates, $R_{g}$ agg, were obtained from the scattering intensity provided by the slow mode according to the following relation: ${ }^{2}$

$$
P(q)=1-\frac{1}{3} R_{g a g g}^{2} q^{2}
$$

where $P(q)$ is the particle scattering factor, which represents the ratio of the scattering light intensity at wave vector $q$ to the zero-wave-vector scattering light intensity.

The polymer volume fraction, $\varphi_{a g g}$, in the aggregates was estimated by the following formula under the assumption that the aggregates can be regarded as homogeneous spheres

$$
\phi_{\text {agg }}=\frac{\bar{v} M_{a g g}}{N_{A}(4 \pi / 3) R_{g a g g}^{3}}
$$

where $\bar{v}$ is the specific volume of chitosan, $M_{\text {agg }}$ is the molecular weight of the aggregates, and $R_{g \text { agg }}$ is their gyration radius.

Determination of Specific Volume. A 2 g/L stock solution of chitosan was prepared by weighing out a dry powder of chitosan and dissolving it in a solvent (aqueous solution containing $0.3 \mathrm{M}$ acetic acid and $0.05 \mathrm{M}$ sodium acetate) preliminary filtered through a $0.22-\mu \mathrm{m}$ Millipore Millex-LCR filter. Solutions of lower concentrations were obtained by diluting the stock solution of chitosan with filtered solvent. For density measurements, a pycnometer calibrated with distilled water was used. First, the empty pycnometer was weighted and then filled with solvent (or solution of chitosan). Then the filled pycnometer was thermostated in a water bath at 25.0 $\pm 0.1{ }^{\circ} \mathrm{C}$ and the volume of liquid inside it was determined. Specific volume $\bar{v}$ of chitosan was calculated using the following equation: 


$$
\bar{v}=\frac{1-\frac{d \rho}{d c}}{\rho_{0}}
$$

where $\rho_{0}$ is the density at infinite dilution (i.e. the density of pure solvent) and $d \rho / d c$ is the density increment, which is obtained from the slope of the dependence of solution density versus polymer concentration (Figure S1). The value of specific volume was found to be equal to $0.570 \pm 0.013 \mathrm{~mL} / \mathrm{g}$.

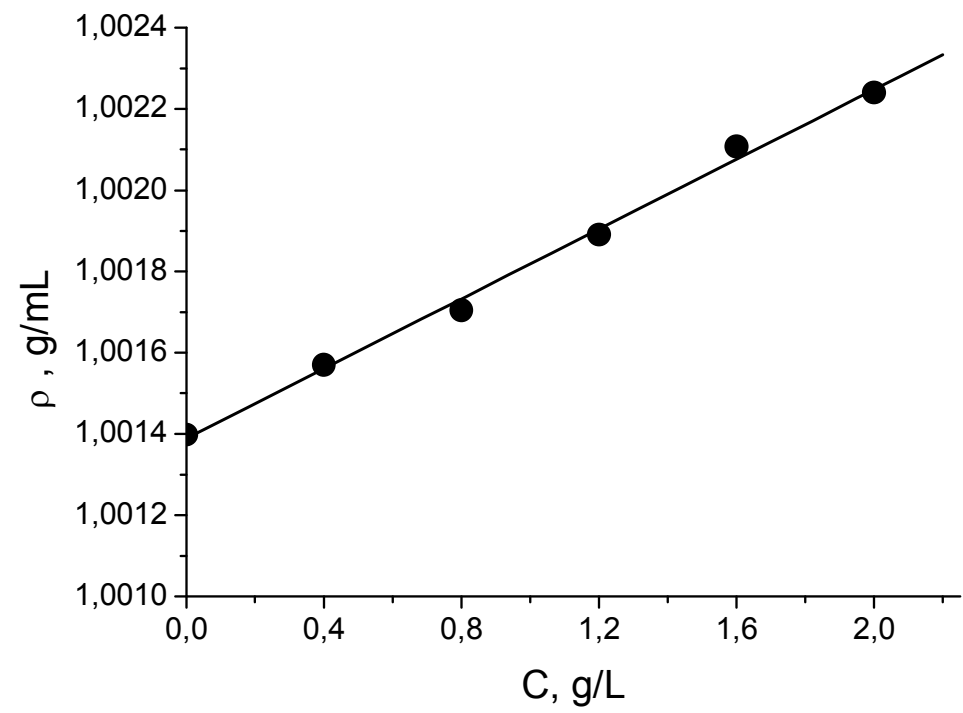

Figure S1. Dependence of the density of chitosan solution on the concentration of polymer at $25^{\circ} \mathrm{C}$. Solvent: aqueous solution containing $0.3 \mathrm{M}$ acetic acid and $0.05 \mathrm{M}$ sodium acetate.

\section{References}

(1) Schartl, W. Light Scattering from Polymer Solutions and Particles Dispersions. Springer: Verlag Berlin Heidelberg, 2007.

(2) Zimm, B. H. J. Chem. Phys. 1948, 16, 1099-1116.

(3) Provencher, S. W. Macromol. Chem. 1979, 180, 201-209. 